\title{
Towards the Applications of Algorithms for Inverse Solutions in EEG Brain-Computer Interfaces
}

\author{
Urszula Jagodzińska
}

\begin{abstract}
Locating the sources of EEG signals (signal generators), i.e. indicating the places in the brain that the signals come from is the objective of the inverse problem in BCI applications using EEG. The two algorithms based on the methods used in the inverse problem: the linear least squares method and the LORETA $^{1}$ method were compared. An analysis of the accuracy of locating the sources generating EEG signals on the basis of the two above mentioned methods was carried out with the use of the MATLAB programme. The findings made it possible to determine both the complexity of calculation involved in the methods under consideration and to compare the accuracy of the results obtained. Tests were done in which the inverse problem was solved on the basis of the data that were entered from the electrodes. Then potentials on electrodes were found by means of solving the forward problem once again $\Phi(\Phi \longrightarrow \widehat{\Phi})$. Moreover, tests were conducted on simulated data describing current density at selected places in the brain. In this case potentials on the electrodes were found by means of solving the forward problem. Subsequently the inverse problem was solved and potentials at selected places in the brain were specified $J(J \longrightarrow \widehat{J})$. In the case of $J(J \longrightarrow \widehat{J})$ only the relative error was examined, while the variance was studied in both cases. As a result of doing the tests, it was proved that relative errors were the same in the SVD and PINV methods, while in the LORETA method the error was similar. The variance computed for these methods was more differentiated for each of the cases, which made it possible to compare the algorithms in a better way. Differentiation of the variances under 0.2 shows that the algorithms that have been analyzed work properly.

On the basis of knowing the results of the inverse problem, an attempt was made to make a selection of the best features of the EEG signal which differentiates the classes. In the present work tests were conducted to examine the differentiation of selected classes. Welch's t-statistics was used to differentiate and order them. The results of the tests present the order for three classes of thought tasks, i.e. imagining moving one's left hand, imagining moving one's right hand, imagining generating words beginning with a randomly chosen letter. The present work is an introduction to a wider classification of features which are made with the use of inverse solutions.
\end{abstract}

Keywords-BCI, EEG, inverse solutions, minimizing norm method, LORETA

\section{INTRODUCTION}

A CCORDING to the definition coined by Jonathan $\mathrm{R}$. Wolpaw at the first international conference on the brain-computer interface (BCI) in 1999 r. "A brain-computer interface $(\mathrm{BCI})$ is a communication or control system in which the user's messages or commands do not depend on

U. Jagodzińska is with Bumar Elektronika S.A., Poligonowa 30, 04-051 Warszawa, Poland (e-mail: urszula.jagodzinska@bumar.com).

${ }^{1}$ The LORETA method and the inverse problem with the algorithms used for calculation were presented in the article [1]. the brain's normal output channel. That is, the message is not carried by nerves and muscles, and, furthermore, neuromuscular activity is not needed to produce the activity that does carry the message...". The electromagnetic waves of the brain are registered by means of electroencephalography (EEG) or magnetoencephalography (MEG) techniques. In BCI it is EEG, which examines the electrical activity of the brain, that is most frequently used. It is on this technique that the present article focuses. The electrical EEG signal is recorded from the surface of the scalp by means of electrodes. Some features of the registered signal are used in BCI systems. These signals are classified and interpreted in real time, e.g. for steering. Locating the sources of the brain's activity registered on the surface of the scalp in space, i.e. solving the inverse problem, is one of the basic problems in BCI. Since work is conducted on the simplified model of the sources perceived as current dipoles placed at different points of the brain, it must be remembered that there is an infinite number of different configurations of these sources which generate the same distribution of potentials on the surface of the scalp. Effective algorihtms for inverse methods can influence the reduction of errors while locating signal sources. Using inverse solutions can improve the classification of EEG signals. The first stage leading to solving the inverse problem is to formulate the forward problem. This is done by means of computing the distribution of the potentials placed on the surface of the head. It is assumed that the distribution of current density inside the brain is known. The space of the brain is divided into hypothetical elements of space - i.e. voxels. The direction and value of current density in each voxel is assumed to be constant. For each voxel these values are represented by the $J$ vector which is three times as large as the number of voxels (three space coordinates). The potentials measured on the electrodes on the surface of the scalp are recorded on the $\Phi$ vector whose measurements are equal to the number of electrodes [2].

The article presents and compares computation algorithms which are indispensable for solving inverse problems. This is based on locating the places in the brain that the EEG signals come from. They are read by means of electrodes placed on the surface of the head. The author's algorithms were presented. Subsequently their accuracy was tested using preprocessed data, including those provided by the Idiap Research Institute. In this study the spherical head model was adopted with the scull surface radius of $R=80 \mathrm{~mm}$. It was assumed that the center of the head model is located at a certain point inside the head and the radius of the sphere with the cerebral cortex equals $r=60 \mathrm{~mm}$. 
Examples of the application of inverse problems can be found in the following works: [3], [4], [5], [6].

In the present article all the $n$-element vectors presented are called matrices with the measurements $n \times 1$.

\section{Presentation of the Problem and Proposed SOLUTIONS}

Solving the inverse problem consists in specifying the location of the EEG sources (generators). This means that the places that the signals come from are designated [1].

Forward problem:

$$
\Phi=K J
$$

where $\Phi$ is the matrix measuring $n_{e} \times 1$, with the $n_{e}$ number of electrodes placed on the surface of the head. The potentials measured at the electrodes (sensors) are recorded in the $\Phi$ matrix and $J=\left(j_{1}, j_{2}, \ldots, j_{n_{v}}\right)^{T}$ is the matrix measuring $\left(3 \cdot n_{v}\right) \times 1$ where $j_{\beta}=\left(j_{\beta_{x}}, j_{\beta_{y}}, j_{\beta_{z}}\right)^{T}$, while $\beta=1, \ldots, n_{v}$ is the matrix of current density in each particular voxel $\beta$. The strength of the source and its location are important in the inverse problem. Let $K$ be the matrix of measurement $n_{e} \times\left(3 n_{v}\right)$. The $K$ matrix matches individual electrodes with voxels. Usually $n_{v}>>n_{e}$. The $K$ matrix is called the transfer matrix [1].

Inverse problem:

$$
\widehat{J}=T \Phi
$$

where $\widehat{J}$ approximation of the $J$ matrix, $T$ is the generalized inverse matrix. The solution to the inverse problem $\widehat{J}$ is, therefore, only an approximation to the $J$ matrix. The extent of the error will depend on the $T$ matrix. The structure of the $T$ matrix depends on the method of solving the inverse problem.

The algorithms that have been suggested for solving inverse problems:

1) Minimizing the norm using the method of least squares (PINV). Let us now look for the min

$$
\min _{J}\|\Phi-K J\|^{2}
$$

where $\|\bullet\|$ denotes the Euclidean norm. In order to specify the minimum of the norm [7] the minimum of function $F(J)=\|\Phi-K J\|^{2}$ is examined. The minimum that is looked for is determined in the $\widehat{J}$ matrix, which is the solution to the so-called normal equation $\left(K^{T} K\right) \widehat{J}=K^{T} \Phi$. The solution to the normal equation takes the form

$$
\widehat{J}=\left(K^{T} K\right)^{-1} K^{T} \Phi
$$

The $\left(K^{T} K\right)^{-1} K^{T}$ matrix is the Moore-Penrose pseudoinverse matrix $K^{+}$. Let mark $\left(K^{T} K\right)^{-1} K^{T}=K^{+}$. Hence

$$
\widehat{J}=K^{+} \Phi, \quad T=K^{+}
$$

The $K^{+}$matrix can by determined using the MATLAB programme.
2) Minimizing the $\|\Phi-K J\|^{2}$ norm using QR decomposition of the $K$ matrix [8] (QR).

$$
K=Q R P^{T}
$$

where tha $Q$ matrix and the $P$ matrix are orthogonal matrices. $Q$ is measuring $n_{e} \times n_{e}, P$ is the permutation matrix (orthogonal) $\left(3 n_{v}\right) \times\left(3 n_{v}\right), R=[A \mid B]$, $A_{n_{e} \times n_{e}}$, rank $A=n_{e}$. Based on [8], supposing $B=[0]_{n_{e} \times\left(3 n_{v}-n_{e}\right)}$ it is possible to minimize norm $\|\Phi-K J\|^{2}$

$$
\left.\widehat{J}=P \cdot\left[\frac{A^{-1} Q^{T} \Phi}{\mathbf{0}}\right]\right\}\left(3 n_{v}-n_{e}\right) \times 1
$$

In this case only the limited number $n_{e}$ voxels, relating only to the linearly independent columns of the $K$ matrix were taken into account. This is a significant limitation of the algorithm presented, since it was possible to analyse only selected voxels in calculating the error.

3) Minimizing the $\|\Phi-K J\|^{2}$ norm using the SVD decomposition of the $K$ matrix [8] (SVD).

$$
K=U S V^{T}
$$

where $U$ is the orthogonal matrix $n_{e} \times n_{e}, V_{\left(3 n_{v}\right) \times\left(3 n_{v}\right)}$ is the orthogonal matrix, $S_{n_{e} \times\left(3 n_{v}\right)}$ as $[C \mid \mathbf{0}]$, where $C$ is the diagonal matrix. The pseudoinverse $S^{+}$matrix can be obtained by taking the inverse elements on the diagonal of the matrix $C$. Then the $K J=\Phi$ equation takes the form $U S V^{T} J=\Phi$, therefore

$$
\widehat{J}=V S^{+} U^{T} \Phi
$$

is the solution to the inverse problem, where $V S^{+} U^{T}=T$.

4) The LORETA method (LORETA). The method was presented in the following articles: [9], [10], [11]. The article [1] presents one version of this method. It consists in finding the extremum

$$
\min _{J}\left(J^{T} W J\right) \text {, under constraint } \Phi=K J
$$

where $W$ is a positive defined weight matrix. The approximate solution $J$ then takes the form

$$
\widehat{J}=T \Phi
$$

where

$$
T=W^{-1} K^{T}\left[K W^{-1} K^{T}\right]^{+}
$$

\section{DESCRIPTION OF THE RESUlts OBtAined}

In order to check that the algorithms described in the previous chapter work correctly, tests were conducted using the MATLAB programme. The calculations were based on the assumption that the way the electrodes were placed on the surface of the head is consistent with the 10-20 (the 
standard recommended by the International Federation of Clinical Neurophysiology IFCN [12]). The electrode signals that were used were taken from the signal database made available by the Idiap Research Institute ${ }^{2}$. In addition, numbers obtained in a random sampling mode were used. Their Cartesian coordinates were applied for points specifying the location of the electrodes and voxels (based on their spherical coordinates). All the calculations were made according to the Idiap assumptions, i.e. 32 electrodes were taken into account: Fp1, AF3, F7, F3, FC1, FC5, T7, C3, CP1, CP5, P7, P3, Pz, PO3, O1, Oz, O2, PO4, P4, P8, CP6, CP2, C4, T8, FC6, FC2, $\mathrm{F} 4, \mathrm{~F} 8, \mathrm{AF} 4, \mathrm{Fp} 2, \mathrm{Fz}, \mathrm{Cz}$, while in the case of preprocessed data, the $\mathrm{C} 3, \mathrm{Cz}, \mathrm{C} 4, \mathrm{CP} 1, \mathrm{CP} 2, \mathrm{P} 3, \mathrm{Pz}$ and $\mathrm{P} 4$ electrodes were examined.

In the spherical model of the head that was assumed, the $\phi=20^{\circ}, \Theta=10^{\circ}$ spherical coordinates were adapted for calculation, which produced 162 voxels. The view of the voxels and the 32 electrodes is presented in Fig. $1^{3}$ and Fig $2^{4}$. The Cartesian coordinates of the placement of both the electrodes and the voxels were calculated by the author on the basis of the spherical coordinates of these points. The voxels were marked blue and the electrodes red.

Data set $\mathrm{V}$ featuring the following parameters: 3 classes, 32 EEG channels (DC-256 Hz), $512 \mathrm{~Hz}$ sampling rate, continuous EEG and precomputed features ${ }^{5}$ (multi-class problems, classification of continuous EEG without trial structure) were taken from the signal database made available by Idiap and prepared for the BCI Competition III [13]. The V packet that was used contains data referring to the performance of thinking tasks at a specified time by three users. The people examined performed the following tasks in a continuous way: imagining moving one's left hand, imagining moving one's right hand, imagining generating words beginning with a randomly chosen letter. The packet included both raw and preprocessed signals. The processing of the data consisted of performing Laplace's space filtration and subsequently Power Spectral Density (PSD) at the frequency domain $8-30 \mathrm{~Hz}$.

In order to check that the algorithms work correctly for a sample of arbitrarily recorded random data and for ordered data, the $\langle 0,1\rangle$ interval was tested both when simulating the recording of potentials for the electrodes and for the voxels. In the case of recording ordered data, the layout of the areas was assumed according to Figs. 3 and 4.

Figure 3 shows examples of ordering the areas of the data recorded into the electrodes in order to check that the calculation algorithms based on the linear least squares method and the LORETA method work correctly for the implemented calculation methods.

An example of ordering the areas of the data recorded into voxels in order to check that the calculation algorithms implemented work correctly are shown in Fig. 4.

In the case of the recorded data (raw and ordered), a single moment of time was considered, while in the case of Idiap data

\footnotetext{
${ }^{2}$ Idiap Research Institute Switzerland (Silvia Chiappa, José del R. Millán).

${ }^{3}$ Created by the Author with the use of MATLAB.

${ }^{4}$ Created by the Author with the use of MATLAB.

${ }^{5}$ More information on Data Set $\mathrm{V}$ on the web page http://www.bbci.de/ competition/iii/desc_V.html.
}

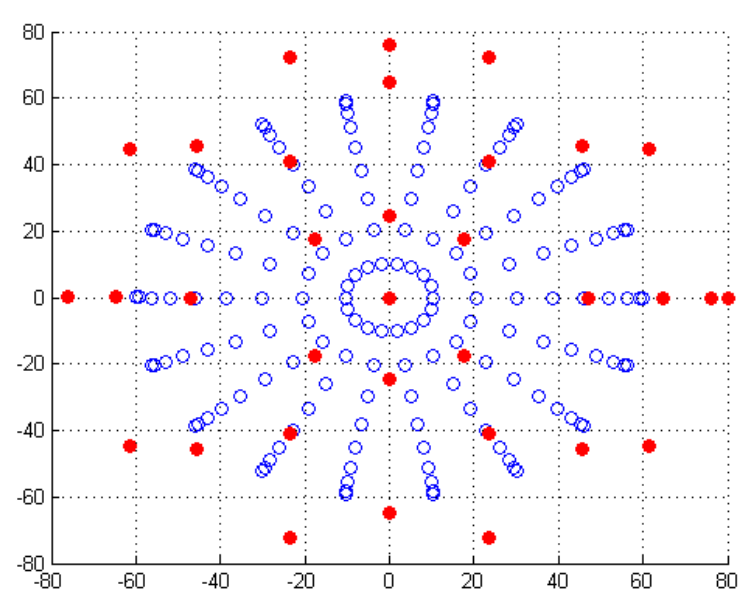

Fig. 1. The map of the voxels and the electrodes - view of the top.

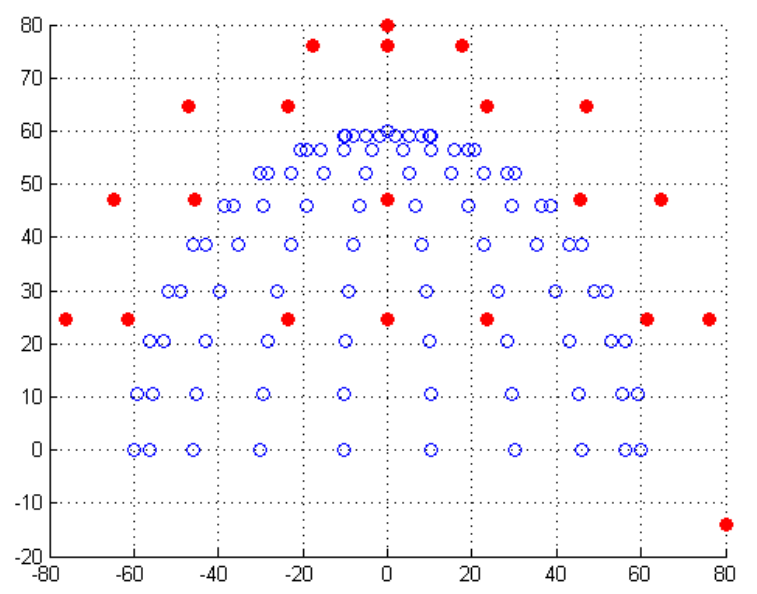

Fig. 2. The map of the voxels and the electrodes - view of the side.
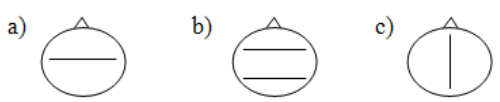

d)
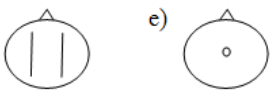

Fig. 3. Examples of ordering the areas of the data recorded into the electrodes $(a, b, c, d, e)$.

the algorithm worked for all the registered moments of time in turn. Results were presented at $10 \mathrm{~Hz}$ frequencies (Alpha and $\mathrm{Mu}$ wave frequencies), and $20 \mathrm{~Hz}$ (Beta wave frequencies) for preprocessed Idiap data.

Results obtained for various methods were compared. In the case where data were recorded into voxels $(J \longrightarrow \widehat{J})$ in order to calculate the error the calculation included:

- the relative error

$$
\frac{\sum_{k}\left(\widehat{J}_{k}-J_{k}\right)^{2}}{\sum_{k}\left(J_{k}\right)^{2}}
$$

$k$ runs from 1 to $n_{v}, J_{k}$ is the exact solution of the problem $\Phi=K J, \widehat{J}$ is approximation $J$, where $\widehat{J}_{k}=\sqrt{\widehat{J}_{k x}^{2}+\widehat{J}_{k y}^{2}+\widehat{J}_{k z}^{2}}$ and $J_{k}=\sqrt{J_{k x}^{2}+J_{k y}^{2}+J_{k z}^{2}}$ 


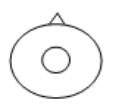

Fig. 4. Example of ordering the areas of the data recorded into the voxels.

- variance

$$
\frac{\sum_{k}\left(\widehat{J}_{k}-\bar{J}\right)^{2}}{n_{v}}
$$

where $\bar{J}=\frac{1}{n_{v}} \sum_{k} J_{k}, k$ runs from 1 to $n_{v}$.

Table I presents the relative error and the variance for the recorded $J(J \longrightarrow \widehat{J})$. The value of the relative error is given in dimensionless quantities, while the variance unit is $\left(A / m^{3}\right)^{2}$.

TABLE I

RELATIVE ERROR AND VARIANCE FOR $J(J \longrightarrow \widehat{J})$ RECORDS

\begin{tabular}{|l|cc|cc|}
\hline \multirow{2}{*}{ Method } & \multicolumn{2}{|c|}{ ordered } & \multicolumn{2}{c|}{ random } \\
\cline { 2 - 5 } & relative error & variance & relative error & variance \\
\hline SVD $^{\mathrm{a}}$ & 0.1344 & 0.0281 & 0.0783 & 0.0229 \\
PINV $^{\mathrm{b}}$ & 0.1344 & 0.0281 & 0.0783 & 0.0229 \\
$\mathrm{QR}^{\mathrm{c}}$ & - & - & - & - \\
LORETA & 0.1433 & 0.0310 & 0.0819 & 0.0223 \\
\hline
\end{tabular}

a calculations for the algorithm with the SVD layout

b calculations for the Moore-Penrose pseudo inverse algorithm

c calculations for the algorithm with the QR layout

In the case of the QR method, the relative error and the variance were not tested, due to the specific nature of the algorithm. The QR algorithm should be treated as one of the proposal of solving the inverse problem.

In the case of recording data into electrodes $\Phi(\Phi \longrightarrow \widehat{\Phi})$ in order to calculate the error the variance can be calculated as

$$
\frac{\sum_{k}\left(\widehat{\Phi}_{k}-\bar{\Phi}\right)^{2}}{n_{e}}
$$

where $\bar{\Phi}=\frac{1}{n_{e}} \sum_{k} \Phi_{k}, k$ runs from 1 to $n_{e}, \widehat{\Phi}$ is the approximation of $\Phi(\widehat{\Phi}=K \widehat{J}, \widehat{J}=T \Phi$, where $T$ is the generalized matrix which is an inverse of the $K$ matrix). The variance values obtained in the case of the $\Phi(\Phi \longrightarrow \widehat{\Phi})$ supplied with Idiap data, arbitrarily recorded random data and ordered data are the same for all the methods of computing (the minimum of squares (SVD, PINV, QR) and LORETA) depending on the case under consideration.

Regarding the ordered $\Phi$ values presented in Fig. 3 they are as follows: a) variance $=0.0886, \mathrm{~b}$ ) variance $=0.0590, \mathrm{c}$ ) variance $=0.0048, \mathrm{~d}$ ) variance $=0.1594, \mathrm{e}$ ) variance $=0.0675$. In the case of random $\Phi$ the variance obtained for all the calculation methods is 0.0779 . As for $\Phi$ Idiap data the $10 \mathrm{~Hz}$ variance $=0.00161$, for $20 \mathrm{~Hz}$ the variance $=0.00004$.

The present paper gives the mean variance for all the moments of time for Idiap data (because of a large number of Idiap data). The low values of variance when using real Idiap data show that the calculation algorithms are accurate. It is significant that in all the cases the $\mathrm{QR}$ algorithm chooses only linearly independent voxels. Due to this the information obtained as a result of using the algorithm seems to be clearer, but its downside is that the information is not complete.
The LORETA method is characterized by a much longer time of calculation than the linear least squares method. This is probably due to the greater complexity of the LORETA method when compared to the linear least squares method. Due to this the LORETA algorithm is more difficult when it comes to implementing it in real time. After analyzing the results obtained and their accuracy, it was ascertained that the rendering of the location is possible only after preprocessing. Implementing the calculation algorithm on the basis of raw data read from the electrodes (Raw) without preprocessing the signal does not yield correct information on the source of the signal. Both recorded ordered and random data can also be treated as raw data (Raw). Obtaining reliable information on the location of the source is possible only by means of analyzing the data that were preprocessed. The functioning of the algorithms on preprocessed data for the Alpha, Beta and $\mathrm{Mu}$ rhythms was analyzed. Significant variation of the values of the potential was observed in voxels connected with mental activity in the motor cortex (connected with changes in the Beta and $\mathrm{Mu}$ waves). There was observed attenuation of potential in voxels connected with mental activity in the occipital lobe area (connected with Alpha waves). Besides calculating the variances checking the calculation of the computing algorithms for Idiap data, a comparison of the sources designated by means of the author's own calculations were compared to those specified by the generally accessible sLORETA program, whose author is Pascual-Marqui R.D. The characteristic feature of that program is that it is known for its good location results. The layout of the areas located as sources of EEG signals was similar both in case of using the author's own calculation algorithms and the program by Pascual-Marqui R.D.

\section{ANALYSIS AND INTERPRETATION OF EEG SignALS}

Knowing the location of the electrical signals of the brain obtained when finding the solution to the inverse problem can help analyze and interpret EEG signals. The diagram (Fig. 5) shown in paper [14] p. 89 can be an inspiration for research on using inverse problems in BCI.

The current article presents an introductory selection of features on the basis of locating generators and describes the attempt that was made to classify those features. It is known

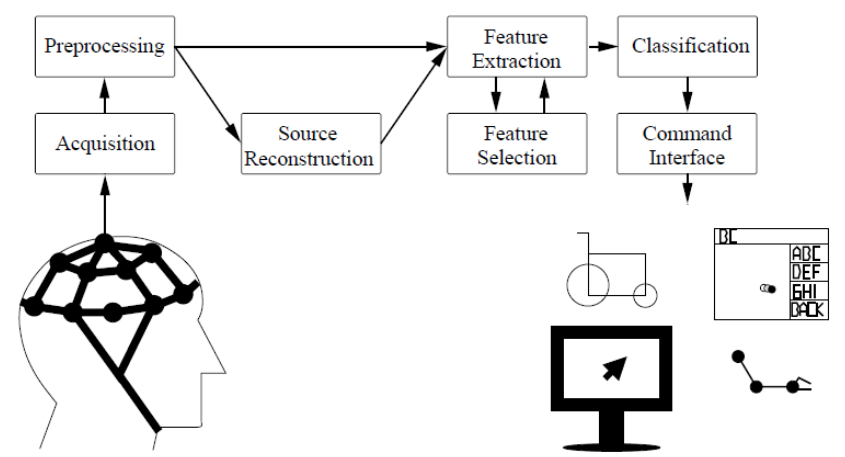

Fig. 5. Inverse problem in BCI. [14] 
that the number of features generated by the brain for thinking tasks ranges from a few to a few thousand [15]. The large number of features makes it impossible to teach the classifier in an effective way. The paper [15] suggests using t-statistics to order and reduce the features to be used in BCI systems.

The present article suggests using such statistics to conduct the test. So that t-statistics is specified with the formula

$$
t=\frac{\bar{x}_{1}-\bar{x}_{2}}{\sqrt{\frac{S_{1}^{2}}{N_{1}}+\frac{S_{2}^{2}}{N_{2}}}}
$$

where $\bar{x}_{i}$ is the approximation in the $i$-sample, $S_{i}^{2}$ is a variance in the $i$-sample, $N_{i}$ is the size of the sample, for $i=1,2$. The value of t-statistics for two samples specifies the degree of differentiation of the features in two different samples. An experiment was conducted comprising in testing the hypotheses using this statistics at the assumed level of significance. Since solutions to the inverse problem were calculated for the purpose of this experiment, the test relates to those solutions, and not the potentials on the electrode like in [15].

Description of the experiment. The signals which were used for the experiment were taken from the Idiap Research Institute database. Besides, information on the location of signals generated by brain neurons were used. This information can be obtained if one knows the elements of the $\widehat{J}$ matrix measuring $3 n_{v} \times 1$ (formulas (4), (7), (9), (11)), where every element of the matrix takes the form $\widehat{J}_{\beta}=\left(\widehat{J}_{\beta_{x}}, \widehat{J}_{\beta_{y}}, \widehat{J}_{\beta_{z}}\right)^{T}$, $\beta=1, \ldots, n_{v}$, where $n_{v}$ is the number of the voxels under consideration. The length $\left|\widehat{J}_{\beta}\right|=\sqrt{\left(\widehat{J}_{\beta_{x}}^{2}+\widehat{J}_{\beta_{y}}^{2}+\widehat{J}_{\beta_{z}}^{2}\right)}$ of the vector can also be calculated. Besides, the Cartesian coordinates of the point where the voxel numbered $\beta$ (chapter III) are also known. In the experiment $n_{v}=162$. One sample is a reading of potentials registered by 32 electrodes fixed to the head according to the 10-20 system, while after processing 8 electrodes in the $\Phi$ matrix were taken into account. After solving the inverse problem using the first of the algorithms suggested for the frequency $20 \mathrm{~Hz}$ (Beta wave frequencies), the values that were obtained were recorded in the $\widehat{J}$ matrix. Three classes of thought tasks were taken into account:

- K2 - imagining moving one's left hand

- K3 - imagining moving one's right hand

- K7 - imagining generating words beginning with a randomly chosen letter.

The coordinate features taken into account were voxel coordinates $(\mathrm{x}, \mathrm{y}, \mathrm{z})$ and the density of the current at the voxels $\left(\mathrm{A} / \mathrm{m}^{3}\right)$, i.e. $\left|J_{\beta}\right|$.

Visualization is an important element in selecting the features. The algorithms processed in the course of the experiment can be useful for locating places on the head that signals which are interesting for each class and have the highest current density came from. An example of the visualisation of voxel layout for the left and right hemisphere is presented in Fig. 6. The visualization that was conducted is a result of the calculations that had been made earlier. In each of the three classes $(\mathrm{K} 2, \mathrm{~K} 3, \mathrm{~K} 7)$ it is possible to consider (select) only that part which is significant for the given class of elements in the $\widehat{J}$ matrix connected with the area selected.
304 samples (from class K7), then 272 samples (from class K3) and finally 288 samples (from class K2) were entered in succession. Next, $t$-statistics was used to order the features of the classes under consideration (selected voxels and the current density connected with them). It was assumed that the feature in the $\mathrm{X}$ population being tested is the current density in the voxels which has a normal distribution of the unknown value $\sigma$ in two different populations of thought tasks $(\mathrm{K} 2, \mathrm{~K} 3)$ or $(\mathrm{K} 2, \mathrm{~K} 7)$ or $(\mathrm{K} 7, \mathrm{~K} 3)$. At the significance level of $\alpha=0,05$, the $H_{0}: m_{1}=m_{2}$ hypothesis was verified as opposed to the alternative hypotheses $H_{1}: m_{1} \neq m_{2}$. The size of the sample was in each case over 100, therefore unknown variation can be approximated using $S^{2}{ }_{1}$ and $S^{2}{ }_{2}$. It is then visible that the t-statistics has normal distribution $\mathrm{N}(0.1)$ and the $u(0.025)$ and $u(0.975)$ quantiles can be assumed to be -1.96 and +1.96 respectively. The critical set takes the form $T_{\alpha}=$ $(-\infty,-1.96) \cup(1.96,+\infty)$. The t-statistics values calculated in the experiment are presented in Tab. II.

TABLE II

Statistical VALUES FOR 864 SAMPLES REPRESENTING TESTS REFERRING TO CLASS K7, K3, K2

\begin{tabular}{|l|l|l|}
\hline$(\mathrm{K} 2, \mathrm{~K} 3)$ & $(\mathrm{K} 2, \mathrm{~K} 7)$ & $(\mathrm{K} 7, \mathrm{~K} 3)$ \\
\hline $\mathrm{t}=-34.9$ & $\mathrm{t}=-28.3$ & $\mathrm{t}=-10.2$ \\
\hline
\end{tabular}

It can, therefore, be assumed that in cases 1), 2) and 3) $m_{1} \neq m_{2}$. The conclusion can, therefore, be drawn that in all the cases the differentiation is very large. It can also be seen that the differentiation for $\mathrm{K} 2$ and $\mathrm{K} 3$ is largest.

In order to test the accuracy of the ordering it is also possible to test the $H_{0}$ hypothesis against $H_{1}$ for the subsequent sets of samples of class K2, K3, K7. Sets of samples in the whole Idiap database tested are ordered in such a way that the person tested performed three kinds of tasks requested by the operator (imagination of repetitive self-paced right hand movements (class K3), imagination of repetitive self-paced left hand movements (class K2), generation of words beginning with the same random letter (class K7)). The person being examined changes at random to another task requested by the operator. In the test classes were ordered in the following way: K7, K3, K2, K3, K7, K2, K7, K3, K2, K7, K3, K7. The results provided in Tab. II relate to the initial set of classes $\mathrm{K} 7, \mathrm{~K} 3$, K2.

The whole set of samples was tested in a similar way. In

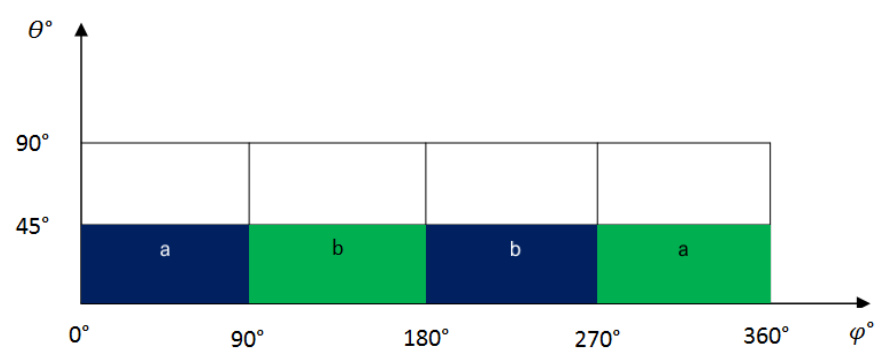

Fig. 6. Example of the visualization of voxel layout for the left and right hemisphere: a) an example of voxel layout in the case of $x<0$ (the left hemisphere), b) an example of voxel layout in the case of $x>0$ (the right hemisphere). 
order to calculate approximate values and variances for the given set of classes, the following formulas were used

$$
\begin{aligned}
\bar{x} & =\frac{1}{N} \sum_{i=1}^{r} \bar{x}_{i} N_{i} \\
S^{2} & =\frac{1}{N} \sum_{i=1}^{r} S_{i}^{2} N_{i}+\frac{1}{N} \sum_{i=1}^{r}\left(\bar{x}_{i}-\bar{x}\right)^{2} N_{i}
\end{aligned}
$$

where $N_{i}$ is the size of the given class $(i=1, \ldots, r)$, $r-$ is the number of classes (set of samples),

$$
\begin{gathered}
N=N_{1}+\cdots+N_{r} \\
\bar{x}_{i}=\frac{1}{N_{i}} \sum_{j=1}^{N_{i}} x_{j} \\
S_{i}^{2}=\frac{1}{N_{i}} \sum_{j=1}^{N i}\left(x_{j}-\overline{x_{i}}\right)^{2} .
\end{gathered}
$$

Table III presents the values of t-statistics calculated for the whole set.

TABLE III

VALUES OF STATISTICS FOR THE WHOLE IDIAP SET CONTAINING SAMPLES REFERRING TO ClASSES K7, K3, K2

\begin{tabular}{|l|l|l|}
\hline$(\mathrm{K} 2, \mathrm{~K} 3)$ & $(\mathrm{K} 2, \mathrm{~K} 7)$ & $(\mathrm{K} 7, \mathrm{~K} 3)$ \\
\hline $\mathrm{t}=-57.3$ & $\mathrm{t}=-25.0$ & $\mathrm{t}=-38.4$ \\
\hline
\end{tabular}

It can be seen that the differentiation between classes $\mathrm{K} 2$ and $\mathrm{K} 3$ is largest. This means there are grounds for using one more test based on [16]. On the basis of testing $k$ samples numbered $N_{1}, \ldots, N_{k} ; N_{1}+\cdots+N_{k}=N$ coming from the $k$ population of unknown mean $m_{1}, \ldots, m_{k}$ and the common $\sigma^{2}$ variance, it is possible to test the $H_{0}: m_{1}=m_{2}=\cdots=m_{k}$ hypothesis (in the experiment being discussed $k=3$ ), $H_{1}$ : not all the mean values are equal.

Let $y_{i j}=\left|\widehat{J}_{j}\right|$, where $j$ are the numbers of selected voxels, $i=1,2, \ldots, k$ are population numbers.

It is possible to conduct Snedecor's F- test.

$$
F=\frac{R_{1}^{2}-R_{0}^{2}}{k} \cdot \frac{N-r}{R_{0}^{2}}
$$

where

$$
\begin{aligned}
R_{1}^{2}-R_{0}^{2} & =\frac{T_{1}^{2}}{N_{1}}+\frac{T_{2}^{2}}{N_{2}}+\cdots+\frac{T_{k}^{2}}{N_{k}}-\frac{T^{2}}{N} \\
T & =T_{1}+\cdots+T_{k} \\
N & =N_{1}+\cdots+N_{k} \\
R_{0}^{2} & =\sum_{i=1}^{k}\left(\min _{m_{i}} \sum_{j}\left(y_{i j}-m_{i}\right)^{2}\right)= \\
& =\sum_{i=1}^{k}\left(\sum_{j} y_{i j}^{2}-\frac{T_{i}^{2}}{N_{i}}\right)
\end{aligned}
$$

The number of degrees of freedom is $\mu_{1}=k-1$ and $\mu_{2}=N-k$; in the experiment described $k=3, k-1=2$. In the test being conducted it is important to assume a common variance $\sigma^{2}$ where there are no reasons to reject the zero hypothesis $H_{0}: \sigma_{1}^{2}=\sigma_{2}^{2}=\cdots=\sigma_{k}^{2}, k \geq 3$. $H_{1}$ : not all the variances are different (Bartlett's test). In order to verify the hypothesis, use was made of statistics, which shows that with the sample size of $N_{i} \geq 6$ for $i=1,2, \ldots, k$ there is $\chi^{2}$ (chi squared) distribution with (k-1) degrees of freedom.

$$
\begin{aligned}
\chi^{2}=\frac{2.303}{c}[ & (N-k) \log \left(\frac{\sum_{i=1}^{k}\left(N_{i}-1\right) S_{i}^{* 2}}{N-k}\right)+ \\
& \left.-\frac{1}{k} \sum_{i=1}^{k}\left(N_{i}-1\right) \sum_{i=1}^{k} \log S_{i}^{* 2}\right]
\end{aligned}
$$

where

$$
\begin{aligned}
\bar{y}_{i} & =\frac{1}{N_{i}} \sum_{j=1}^{N_{i}} y_{i j} \\
S_{i}^{* 2} & =\frac{1}{N_{i}-1} \sum_{i=1}^{k}\left(y_{i j}-\bar{y}_{i}\right)^{2}=\frac{N_{i}}{N_{i}-1} S_{i}^{2} \\
i & =1,2, \ldots, k \\
N & =\sum_{i=1}^{k} N_{i} \\
c & =1+\frac{1}{3(k-1)}\left(\sum_{i=1}^{k} \frac{1}{N_{i}-1}-\frac{1}{N-k}\right)
\end{aligned}
$$

As a result of using the F-statistics test, comparing all the features includes the whole sample.

\section{CONCLUSIONS}

The article presents a comparison of algorithms which are solutions to the inverse problem. Tests were conducted to compute the relative error and variances for the methods discussed in the article used when simulating the entering of data into voxels (arbitrarily recorded random data and ordered data). The relative error does not exceed 0.15 , and the variance is not larger than 0.16 . The variance for the methods under discussion was also computed, i.e. for the least squares method, (PINV, SVD, QR) and LORETA when entering data into electrodes (arbitrarily recorded random data, ordered data and real Idiap data). The lowest error was obtained for real processed data (Idiap). It can be assumed that the location of voxels from which the signals come is only possible on the basis of processed data. Tests were done on the basis of which an introductory selection of features was conducted, i.e. imagining moving one's left hand, imagining moving one's right hand, imagining generating words beginning with a randomly chosen letter. The differentiation of the features examined was obtained on the basis of t-statistics. It was ascertained that the features were generally considerably different, with the greatest differences manifesting themselves in imagining moving one's left hand, imagining moving one's right hand. Moreover, F-Snedecor's statistics was discussed. This method makes it possible to compare the differentiation of all of the features at the same time. Plans have been made to use this statistics in future research. The article also suggests that it is possible to compare features in the selected areas described using spherical coordinates of the 
points belonging to these areas, as shown in Fig. 6. Besides, there are plans for improving the effectiveness of calculation algorithms for inverse methods and developing an authored algorithm for locating sources, their strength and direction. Moreover, it is important to focus on increasing the range of preprocessing, which can significantly improve the accuracy of algorithms used for solving inverse problems. Appropriate methods used in preprocessing in connection with choosing appropriate algorithms can influence the reduction of errors while locating generators (signal sources).

\section{ACKNOWLEDGMENT}

Let me express my sincere gratitude to Prof. $\mathrm{PhD}$, hab in engineering Edward Sędek, Bumar Elektronika S.A. and Prof. $\mathrm{PhD}$, hab Wiesław Sasin, head of the Differential Geometry Department at the Faculty of Mathematics and Information Science, the Warsaw University of Technology.

\section{REFERENCES}

[1] U. Jagodzinska and L. Oskwarek, "Low resolution electromagnetic tomography method as an example of solving the inverse problem in brain computer interface," ELEKTRONIKA, pp. 89-91, 2011.

[2] — " "Metoda LORETA jako przykład metody rozwiazywania zagadnienia odwrotnego w interfejsie mózg-komputer ("Low resolution electromagnetic tomography method as an example of solving the inverse problem in brain computer interface")," in 5 Konferencja Urzadzenia i Systemy Radioelektroniczne (UiSR) DNI RADIOLOKACJI 2012 in SOBIENIE SZLACHECKIE, 6-7 December 2012.

[3] M. Congedo, F. Lotte, and A. Lecuyer, "Classification of movement intention by spatially filtered electromagnetic inverse solutions," Physics in Medicine and Biology, vol. 51, pp. 1971-1989, 2006.

[4] R. G. de Paralta Menedez, S. G. Andino, L. Perez, P. W. Ferrez, and J. D. R. Millan, "Non-invasive estimation of local field potentials for neuroprosthesis control," Cognitive Processing, vol. 6, pp. 59-64, 2005.
[5] B. Kamousi, Z. Liu, and B. He, "Classification of Motor Imagery Tasks for Brain-Computer Interface Applications by Means of Two Equivalent Dipoles Analysis," IEEE Transactions On Neural Systems And Rehabilitation Engineering, vol. 13, no. 2, pp. 166-171, June 2005.

[6] L. Qin, L. Ding, and B. He, "Motor imagery classification by means of source analysis for brain computer interface applications," Journal of Neural Engineering, vol. 1, pp. 133-141, 2004.

[7] U. Jagodzinska, "The implementation of algorithms for inverse solutions in eeg brain-computer interfaces," in Conference materials, Signal Processing Symposium (SPS-2013) in Jachranka, June 2013.

[8] C. L. Lawson and R. J. Hanson, Solving Least Squares Problems. Classics in Applied Mathematics, Society for Industrial and Applied Mathematics Philadelphia SIAM, 1995.

[9] A. Painold [and others], EEG low-resolution brain electromagnetic tomography (LORETA) in Huntington's disease. Springer-Verlag, 2010 , published online: 12 December 2010.

[10] R. D. Pascual-Marqui, "Review of Methods for solving the EEG Inverse Problem," International Journal of Bioelectromagnetism, vol. 1, no. 1, pp. 75-86, 1999.

[11] , Discrete, 3D distributed linear imaging methods of electric neuronal activity. Part 1: exact, zero error localization. Zurich, Switzerland: The KEY Institute for Brain-Mind Research University Hospital of Psychiatry, 17 October 2007.

[12] H. Jasper, "The ten-twenty electrode system of the International Federation," Electroencephalogr Clin Neurophysiol, vol. 10, pp. 371-375, 1958.

[13] B. Blankertz, K.-R. Muller, G. Curio, T. M. Vaughan, G. Schalk, J. R. Wolpaw, A. Schlogl, C. Neuper, G. Pfurtscheller, T. Hinterberger, M. Schroder, and N. Birbaumer, "The BCI competition 2003: Progress and perspectives in detection and discrimination of EEG single trials," IEEE Transactions on Biomedical Engineering, vol. 51, no. 6, pp. 1044 1051, June 2004.

[14] Q. Noirhomme, "Localization of Brain Functions: Stimuling Brain Activity and Source Reconstruction for Classification," in These presentee en vue de l'obtention du grande de Docteur en Science Appliquees. Universitate Catholique de Louvain, October 2006.

[15] M. Kołodziej, A. Majkowski, and R. J. Rak, "Application of t-statistics for processing of EEG signal in brain-computer interface," Przeglad Elektrotechniczny, vol. 9a, pp. 187-189, 2011.

[16] C. R. Rao, Modele liniowe statystyki matematycznej. PWN, 1982 ("Linear Statistical Inference and its applications", J. Wiley \& Sons, 1973). 